\title{
Evaluation of thromboelastometry, thrombin generation and plasma clot lysis time in patients with bleeding of unknown cause: A prospective cohort study
}

\author{
Caroline S. B. Veen ${ }^{1}$ (D) | Elise J. Huisman ${ }^{2}$ | Marjon H. Cnossen ${ }^{2}$ | Regina Kom-Gortat ${ }^{1}$ | \\ Dingeman C. Rijken ${ }^{1}$ | Frank W. G. Leebeek ${ }^{1}$ (D) | Moniek P. M. de Maat ${ }^{1}$ | \\ Marieke J. H. A. Kruip ${ }^{1}$
}

${ }^{1}$ Department of Haematology, Erasmus University Medical Center Rotterdam, Rotterdam, The Netherlands

${ }^{2}$ Department of Paediatric Haematology, Erasmus University Medical Center Sophia Children's Hospital, Rotterdam, The Netherlands

\section{Correspondence}

Marieke J. H. A. Kruip, Department of Haematology, Erasmus MC, University Medical Center Rotterdam, Room Na-808, PO Box 2040, 3000 CA Rotterdam, The Netherlands.

Email: m.kruip@erasmusmc.nl

Funding information

Fonds NutsOhra, Grant/Award Number: 1303-027

\begin{abstract}
Introduction: Diagnostic evaluation of patients with a bleeding tendency remains challenging, as no disorder is identified in approximately $50 \%$ of patients. An impaired interplay of several haemostatic factors might explain bleeding phenotype in these patients.

Objective: To investigate whether global haemostasis assays are able to identify haemostatic abnormalities in patients with a bleeding tendency unexplained by current diagnostic laboratory tests.

Materials and methods: Patients of $\geq 12$ years with a bleeding tendency were included from a tertiary outpatient clinic. Bleeding phenotype was assessed with the ISTH-BAT. Patients were classified as having bleeding of unknown cause (BUC) or a mild bleeding disorder (MBD) based on abnormalities assessed by routine haemostatic tests. Global haemostasis tests (rotational thromboelastometry (ROTEM), thrombin generation test (TG) and plasma clot lysis time (CLT)) were measured in all patients. The results were compared with 76 controls.

Results: One hundred and eighty-one patients were included, and 60\% (109/181) was classified as having BUC. BUC patients demonstrated a significantly prolonged lag time in TG (median 7.7 minutes, IQR 6.7-8.7) and a significantly prolonged CLT (median 60.5 minutes, IQR 54.7-66.1) compared to controls. No differences in ROTEM variables were found. Patients with MBD showed an impaired thrombin generation with a significantly decreased ETP (median $1024 \mathrm{nmol} / \mathrm{L}^{*} \mathrm{~min}$, IQR 776-1355) and peak height (median $95 \mathrm{nmol} / \mathrm{L}, \mathrm{IQR} 76-138$ ), compared to BUC patients and controls. Conclusion: No major differences were found in ROTEM and TG variables in BUC patients compared to controls. BUC patients did have a significantly prolonged clot lysis time. The underlying mechanism for this finding is unknown.
\end{abstract}

KEYWORDS

bleeding, bleeding of unknown cause, fibrinolysis, haemostasis, mild bleeding disorder, thrombin generation, thromboelastometry 


\section{1 | INTRODUCTION}

Patients with a mild bleeding disorder present with varying symptoms, such as easy bruising, mucocutaneous bleeding, and bleeding after surgery or tooth extraction. ${ }^{1}$ However, in the general population bleeding symptoms are reported in more than $20 \%$ of healthy individuals ${ }^{2,3}$ Therefore, diagnostic evaluation of patients with a bleeding disorder is a challenging process. Application of routine diagnostic laboratory tests in patients with clinically relevant bleeding leaves around $50 \%$ of patients without a diagnosis. Subsequently, these patients are classified as patients with bleeding of unknown cause (BUC). ${ }^{4-6}$ Patients with a clinically relevant bleeding phenotype are also regularly diagnosed with mild haemostatic defects which may not sufficiently explain the patient's bleeding phenotype (mild bleeding disorders, MBD). As a clear diagnosis is lacking in these patients, the most appropriate treatment regimen also remains uncertain. ${ }^{7}$

An impaired interplay between several mild haemostatic defects may explain bleeding phenotype in this patient category. Global haemostatic assays may increase insight into the pathogenesis of BUC, as other components of the haemostatic system on blood coagulation are investigated more thoroughly. ${ }^{8}$ Rotational thromboelastometry (ROTEM) provides a graphical representation of blood clot formation and fibrinolysis, which includes contributions of erythrocytes, leucocytes and platelets. ${ }^{9}$ Measurement of thrombin generation (TG) has also been proposed as a promising approach to globally estimate an individual's coagulation potential and to predict a hypoor hyper-coagulable state. ${ }^{10}$ In addition, investigation of fibrinolysis is often omitted in the routine work-up of patients with a bleeding tendency. It is however known that clots made from the plasma of haemophilia patient show altered characteristics and higher susceptibility to fibrinolysis. ${ }^{11,12}$

In order to gain more insight into the pathophysiological mechanisms of bleeding symptoms in patients with BUC and MBD, and to investigate the diagnostic value of global haemostasis tests in these patients, we investigated the role of these global tests in the diagnostic work-up.

\section{2 | MATERIALS AND METHODS}

\section{1 | Study population}

Patients, aged 12 years or older, referred to the outpatient haematology and paediatric haematology clinics of our tertiary clinics, the Erasmus University Medical Center and Sophia Children's Hospital, for haemostatic screening between 1 June 2016 and 1 March 2018 due to a clinically relevant bleeding tendency were prospectively included. Patients previously diagnosed with a bleeding disorder or diagnosed with an established bleeding disorder after a first laboratory panel (eg von Willebrand's disease, haemophilia or platelet disorder), patients using anticoagulant, antiplatelet or nonsteroidal anti-inflammatory drugs, pregnant women and women $<3$ months postpartum were not eligible for study inclusion. A total of 76 sexmatched healthy individuals were included as control group. These healthy individuals were recruited among employees and students of the Erasmus MC University Medical Center. This study was subject to the Medical Research Involving Human Subjects Act and approved by the Medical Ethics Committee of the Erasmus University Medical Center Rotterdam (MEC-2016-218). Written informed consent was obtained from each participant.

\section{2 | Medical bleeding history and bleeding assessment tool}

Upon inclusion, all surgical interventions, tooth extractions, obstetric history and detailed family history were documented. A bleeding score (BS), based on the history of bleeding events, was calculated by the ISTH-Bleeding Assessment Tool (ISTH-BAT), with cut-off values $\geq 4$ in males, $\geq 6$ in females and $\geq 3$ in children. ${ }^{13,14}$

\section{3 | Blood sampling and laboratory assays}

Laboratory tests were performed in a stepwise manner. The first step included a full blood count, $\mathrm{ABO}$ blood type, prothrombin time (PT), activated partial thromboplastin time (aPTT), fibrinogen concentration according to von Clauss, determination of VWF antigen (VWF:Ag), activity (VWF:GPIbM) and collagen binding (VWF:CB), one-stage assay FVIII:C and FIX:C and VWF-multimer analysis in case of low VWF. Platelet function was assessed with the collagenepinephrine and collagen-ADP cartridges on the platelet function analyzer (PFA-200). As second step, FVII:C, FXI:C and FXIII:C, $\alpha 2$-antiplasmin, and light transmission aggregometry (LTA) were performed. Measurements of VWF:Ag/Act/CB and FVIII:C were repeated at least once.

Blood sampling was performed using the Vacutainer system (Becton Dickinson) containing sodium citrate (final concentration $0.109 \mathrm{~mol} / \mathrm{L}$ ) or EDTA (1.8 mg/mL, Plymouth). Citrated blood was centrifuged at $2000 \mathrm{~g}$ for 10 minutes at room temperature, followed by $14000 \mathrm{~g}$ for 10 minutes centrifugation of plasma at room temperature. Platelet poor plasma (PPP) samples were stored in aliquots at $-80^{\circ} \mathrm{C}$ until analysis, when indicated. Routine coagulation tests aPTT (Actin FS), PT (Thromborel S) and fibrinogen (Thrombin Reagent) were measured on a Sysmex CS-5100 (Siemens Healthcare Diagnostics BV). Collagen-ADP (C-ADP) and collagen-epinephrine (C-Epi) cartridges were used to measure closure times (CT, seconds) on the PFA-200 (Siemens). Light transmission aggregometry (LTA) was performed on a Chrono-Log aggregometer 490 (Stago Benelux BV). VWF:Ag levels and VWF:CB activity were determined with an in-house ELISA assay. VWF activity (VWF:GPIbM) was determined with the INNOVANCE VWF Ac assay (Siemens) on a Sysmex CS-5100. FVIII:C and FIX:C were measured using one-stage clotting assays and derived from the prolongation of the clotting time (aPTT) measured on the Sysmex CS-5100 (Siemens). FXIII activity was measured using the Berichrom ${ }^{\circledR}$ FXIII kit 
(Siemens) on the Sysmex CS-5100 (Siemens). Alpha 2-antiplasmin level was measured using a chromogenic assay (Stachrom, Stago) on the Sysmex CS-5100 (Siemens).

\section{4 | Rotational thromboelastometry}

Viscoelastic clotting measurements were performed with ROTEM ${ }^{\circledR}$ Delta (Tem International $\times \mathrm{GmbH}$ ) tests according to the manufacturer's protocol. All investigations were performed within 2 hours after blood collection and the assays ran for 60 minutes. Extrinsic and intrinsic coagulation was measured with the EXTEM and the INTEM assays. The influence of fibrinogen on clot firmness was estimated with the platelet-inactivated FIBTEM assay. The following ROTEM parameters were analysed: clotting time (CT, sec); clot formation time (CFT, sec); maximum clot firmness (MCF, mm); and maximal lysis ( $M L, \%)$.

\section{5 | Thrombin generation}

Thrombin generation was assessed using the calibrated automated thrombogram (CAT) assay (Diagnostica Stago) in accordance with the manufacturers' instructions, as described previously. ${ }^{15,16}$ Briefly, PPP was added to PPP Reagent 1 PM TF (PPP-Reagent LOW, Thrombinoscope BV), which consists of a mixture of tissue factor (TF; 1 PM final concentration in plasma) and phospholipids. Plasma of each subject was analysed in duplicate. Acquisition of thrombin generation parameters was performed using the Thrombinoscope software (Diagnostica Stago; CAT). Four parameters were derived from the thrombin generation curve: lag time (min), time to peak (ttpeak, min), endogenous thrombin potential (ETP, nmol/L*min) and peak height (nmol/L).

\section{6 | Plasma clot lysis assay}

The plasma clot lysis assay was performed as described before. ${ }^{17,18}$ PPP was diluted in buffer $(25 \mathrm{mmol} / \mathrm{L}$ Hepes, $137 \mathrm{mmol} / \mathrm{L} \mathrm{NaCl}$, $3.5 \mathrm{mmol} / \mathrm{L} \mathrm{KCl}, 1 \%(\mathrm{w} / \mathrm{v}) \mathrm{BSA}, \mathrm{pH}$ 7.4). The diluted plasma was added to a reaction mixture, containing tissue factor (TF, Innovin, 1000 times diluted; Dade Behring), $\mathrm{CaCl}_{2}$ (17 mmol/L), tPA (30 ng/mL, Actilyse, Boehringer Ingelheim), phospholipid vesicles (10 $\mu \mathrm{mol} / \mathrm{L}$, Rossix Mölndal) and potato carboxypeptidase inhibitor ( $\mathrm{PCl}$, an inhibitor of activated TAFI) $(30 \mu \mathrm{g} / \mathrm{mL})$ when indicated. The concentrations refer to the final concentrations in the clot. In a microplate reader (Victor ${ }^{\mathrm{TM}}$, PerkinElmer), the optical density at $405 \mathrm{~nm}$ was measured every minute for 300 minutes at $37^{\circ} \mathrm{C}$. The clot lysis time (CLT) was the time from midpoint of minimum turbidity to maximum turbidity, which represents clot formation, to the midpoint of maximum turbidity to minimum turbidity, which represents clot lysis. CLTs with and without the addition of $\mathrm{PCl}$ were measured in duplicate.

\section{7 | Reference ranges}

Reference ranges for ROTEM, thrombin generation and plasma clot lysis time are based on 76 healthy controls, calculated with the Reference Value Advisor software (v2.1) which closely follows the CLSI guideline. ${ }^{19,20}$

\section{8 | Definition of diagnoses}

A MBD was defined as the presence of a hereditary bleeding disorder, specified as follows: low VWF-VWF activity levels between 0.30-0.50 U/mL and ratio of FVIII:C to VWF:Ag > $0.6^{21}$; PFD: abnormalities found using light transmission aggregation testing (LTA), not fitting the pattern of any known platelet function disorder ${ }^{22}$; and isolated coagulation factor deficiency: deficiency of a coagulation factor, other than FVIII (haemophilia A) or FIX (haemophilia B), with laboratory criteria as proposed by the European Network of Rare Bleeding Disorders. ${ }^{6,23}$ Bleeding was considered as bleeding of unknown cause (BUC) based on the absence of haemostatic abnormalities after extensive laboratory investigation, as described before. ${ }^{1,7,24}$

\section{9 | Statistics}

We used descriptive statistics to summarize baseline characteristic of the study population. In case of a skewed distribution, data are presented as median and interquartile range (IQR), and compared by a Mann-Whitney $U$ test. In case of a normal distribution, data are presented as mean and standard deviation (SD) and compared using an independent sample $t$ test. Categorical data are presented as numbers with percentages and compared using a Pearson Chi-square test. In multiple logistic regression, models were adjusted for age, sex, BMI, platelet count, fibrinogen, VWF, FVIII:C and FXIII:C as appropriate. Outcomes are reported as odds ratios (ORs) followed by the $95 \%$ confidence interval $(\mathrm{Cl})$. Multiplicity correction was not performed because of the hypothesis-generating approach of the study. A $P$-value of $<.05$ was considered statistically significant. All analyses were performed with SPSS version 24.0 (IBM, Armonk, NY, USA).

\section{3 | RESULTS}

One hundred and eighty-one patients were referred to our hospital with a clinically relevant bleeding tendency and eligible for inclusion, and 76 healthy individuals were included as healthy controls. The majority of study participants was female ( $84 \%$ of patients and $86 \%$ of healthy controls). Mean age was 33.6 years (SD 17.3) for patients, with $53 / 181$ (29\%) adolescent patients $\geq 12$ years, and 35.8 years (SD 12.3) for healthy controls, see Table 1. For study protocol and flow of inclusion see Figure 1. 
TABLE 1 Study group characteristics

\begin{tabular}{|c|c|c|c|c|c|c|c|}
\hline & \multicolumn{2}{|c|}{$\begin{array}{l}\text { Bleeding of Unknown Cause (BUC) } \\
(n=121)\end{array}$} & $\begin{array}{l}\text { Healthy controls } \\
(\mathrm{HC})(\mathrm{n}=76)\end{array}$ & $P^{c}$ & \multicolumn{2}{|c|}{$\begin{array}{l}\text { Mild bleeding disorder (MBD) } \\
(n=60)\end{array}$} & $P^{d}$ \\
\hline Age, median [IQR] & 33 [24-50] & & $32[26-46]$ & n.s. & 20 [15-39] & & .001 \\
\hline Female, n (\%) & 105 (87\%) & & $65(86 \%)$ & n.s. & 47 (78\%) & & n.s. \\
\hline BMI, median [IQR] & $26.5[22.4-29.8]$ & & $23.6[21.8-27.3]$ & .020 & $24.0[20.3-27.5]$ & & .047 \\
\hline $\begin{array}{l}\text { Abnormal bleeding score }{ }^{a} \text {, } \\
\mathrm{n}(\%)\end{array}$ & 65 (54\%) & & $1(1 \%)$ & .000 & $51(88 \%)$ & & .000 \\
\hline Blood group O, n (\%) & $48(40 \%)$ & & $28(38 \%)$ & .036 & 31 (53\%) & & .049 \\
\hline $\begin{array}{l}\text { Positive family history }{ }^{\mathrm{b}} \text {, } \\
\mathrm{n}(\%)\end{array}$ & $37(31 \%)$ & & $0(0 \%)$ & n.a. & $28(48 \%)$ & & n.s. \\
\hline Presenting symptom, (\%) & Postpartum haemorrhage & $15 \%$ & n.a. & - & Family history & $20 \%$ & - \\
\hline \multirow[t]{3}{*}{ Referring physician, (\%) } & $\begin{array}{l}\text { Haematologist from local } \\
\text { hospital }\end{array}$ & $29 \%$ & \multirow[t]{3}{*}{ n.a. } & \multirow[t]{3}{*}{-} & Paediatrician & $35 \%$ & \multirow[t]{3}{*}{-} \\
\hline & General practitioner & $22 \%$ & & & General practitioner & $24 \%$ & \\
\hline & Gynaecologist & $19 \%$ & & & $\begin{array}{l}\text { Haematologist from local } \\
\text { hospital }\end{array}$ & $22 \%$ & \\
\hline
\end{tabular}

Note: Data are shown as median and interquartile range [25th-75th percentile], and number and percentage, as appropriate.

Abbreviations: BMI, body mass index; BUC, bleeding of unknown cause; HC, healthy controls; MBD, mild bleeding disorder; n.a., not applicable; n.s., nonsignificant.

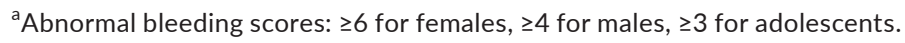

${ }^{b} 1$ st, 2nd or 3rd degree family member diagnosed with a bleeding disorder or evaluated at a haematology outpatient department for a bleeding tendency.

${ }^{\mathrm{C}}$ Comparison of BUC patients and healthy controls.

${ }^{\mathrm{d} C}$ Comparison of BUC patients and patients with a MBD.

A total of $120 / 181$ (66\%) patients were classified as having BUC. Sixty patients were classified as having a MBD, with platelet function disorders (43\%) and low VWF (35\%) being most prevalent. BUC patients consisted of a higher percentage of adults ( $80 \%$ versus $52 \%$ in MBD, $P<.01$ ) and had a higher median age (33 year, IQR 24-50 year) than MBD patients (20 year, IQR 15-39 year, $P<.01)$. Significantly less BUC patients had blood type O (40\%) than MBD patients $(53 \%, P<.05)$. BUC patients had a median BS of 5 (IQR 3-8), compared to a median BS of 7 (IQR 6-9) in patients with MBD $(P<.01)$, with only $54 \%$ of BUC patients presenting with an abnormal BS, compared to $88 \%$ of MBD patients $(P<.01)$ (see Table 1).

As expected, patients with MBD had significantly lower levels of VWF:Ag, VWF:GPIbM, VWF:CB and FVIII:C than BUC patients. Also, MDB patients had a significantly lower platelet count and increased aPTT. No differences in haemostatic variables were found between BUC patients and healthy controls (see Table 2 and Figure 2). When excluding patients with a normal bleeding score, significantly lower levels of VWF:Ag, VWF:GPIbM, VWF:CB, FVIII:C and FIX:C-level were found in MBD patients compared to BUC patients (see Table 2).
When comparing BUC patients and healthy controls, no statistically significant differences were observed in thromboelastometry variables (see Figure 2 and Table S1). When adjusting for age, sex, BMI, platelet count, fibrinogen, VWF:GPIbM and FVIII:C levels by means of logistic regression analysis, no significant differences were found between BUC patients and healthy controls (Table S2). Comparing BUC patients with MBD patients, BUC patients had a significantly decreased clot formation time (CFT) in the EXTEM and INTEM assay, and a significantly increased maximum clot firmness (MCF) in the EXTEM, INTEM and FIBTEM assay (see Figure 2 and Table S1).

BUC patients had a significantly longer lag time (median 7.7 minutes, IQR: 6.6-8.7 minutes) compared to healthy controls (median 6.9 minutes, IQR: 6.0-8.6 minutes, $P<.05$ ). Other thrombin generation parameters were not different between BUC patients and healthy controls (Table S1). When adjusting for age, sex, BMI, platelet count, fibrinogen and FXIII:C levels by means of logistic regression analysis, also no significant differences were found between BUC patients and healthy controls (Table S2). In MBD patients, impaired thrombin generation was found, with a significantly decreased ETP and peak height compared to BUC patients (see Figure 2) and healthy controls. 


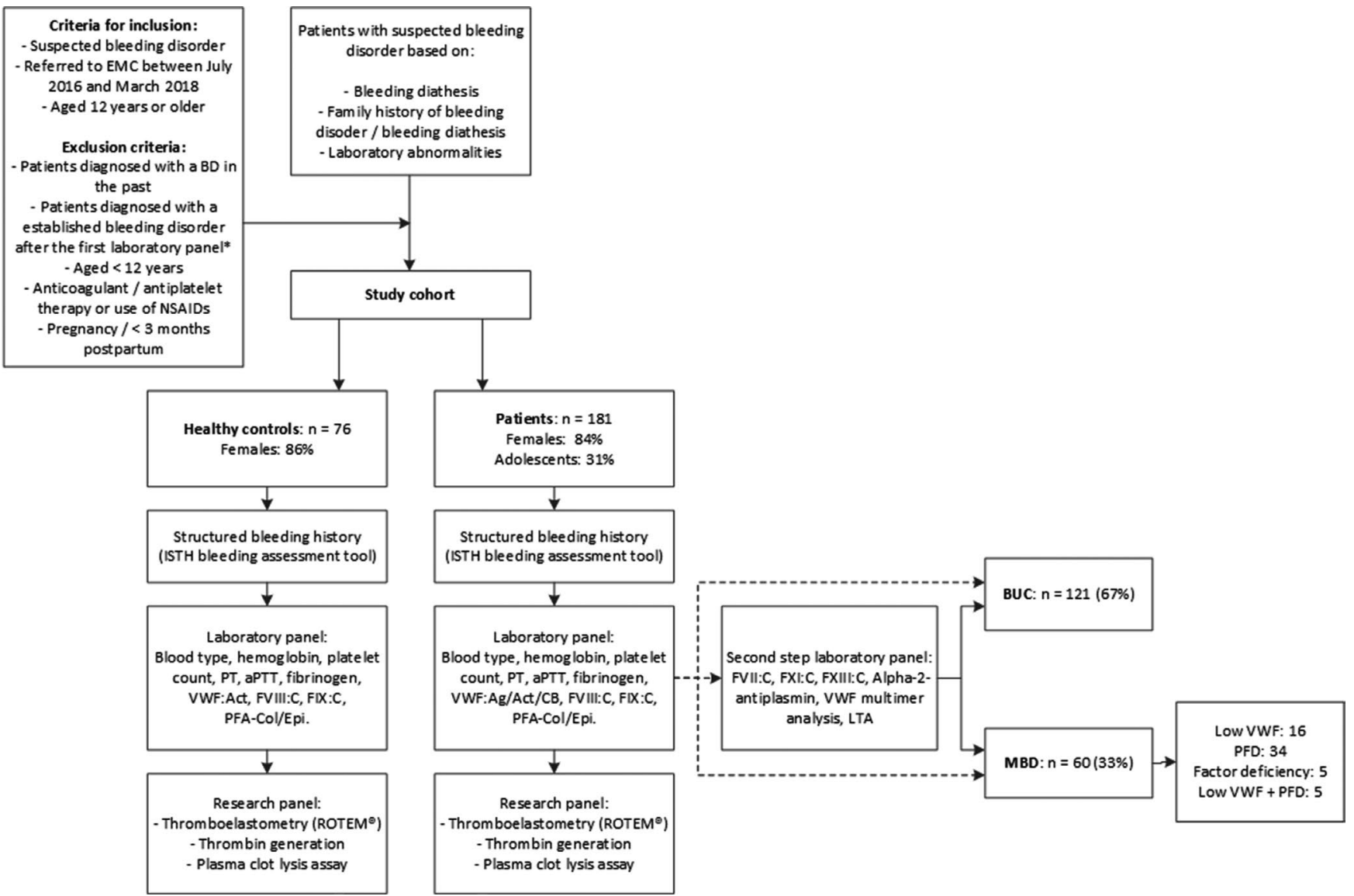

FIGURE 1 Flow chart of study protocol and inclusion. aPTT, activated partial thromboplastin time; BUC, bleeding of unknown cause; FIX, factor IX; FVII, factor VII; FVIII, factor VIII; FXI, factor XI; FXIII, factor XIII; LTA, light transmission aggregometry; MBD, mild bleeding disorder; No BD, no bleeding disorder; PFA-Col/Epi, platelet function analyser collagen/epinephrine; PFD, platelet function disorder; PT, prothrombin time; VWF, von Willebrand factor

Remarkably, a significant longer CLT in BUC patients (PCl- median 60.3 minutes, IQR 54.7-66.0 minutes and $\mathrm{PCI}+41.3$ minutes, IQR 38.0-46.2 minutes) was found compared to healthy controls ( $\mathrm{PCl}$ - median 57.4 minutes, IQR 53.9-61.7 minutes and $\mathrm{PCl}+38.9$, IQR 36.3-42.5 minutes, $P=.03$ and $P<.01$ respectively) (Table $\mathrm{S} 1$ ). However, when adjusting for age, sex, BMI, platelet count, fibrinogen and FXIII:C levels by means of logistic regression analysis, no significant differences were found between BUC patients and healthy controls (Table S2). Overall, no differences were found in CLT between BUC patients and MBD patients, both with and without adjustment for age, sex, BMI, platelet count, fibrinogen and FXIII:C levels.

In thromboelastometry, thrombin generation and clot lysis time variables, no additional significant differences were found between BUC and MBD patients, after excluding patients with a normal bleeding score (see Table 2 and Table S1).

The ETP was significantly lower in patients with an abnormal bleeding score (median $1223 \mathrm{nmol} / \mathrm{L}^{*} \mathrm{~min}, \mathrm{IQR}$ : 923-1516 nmo$\mathrm{I} / \mathrm{L}^{*} \mathrm{~min}$ ) compared to patients with a normal bleeding score (median $1055 \mathrm{nmol} / \mathrm{L}^{*} \min$, IQR: 828-1363 nmol/L*min, $\left.P=.046\right)$. Furthermore, patients with an abnormal bleeding score had a significantly longer CT (median 65 minutes, IQR: 58-72 minutes) in the
EXTEM assay and significantly lower MCF (median $16 \mathrm{~mm}$, IQR: 13-19 $\mathrm{mm}$ ) in the FIBTEM assay compared to patients with a normal score (CT-EXTEM: median 67 minutes, IQR: 63-73 minutes, $P=.048$ and MCF-FIBTEM: median $15 \mathrm{~mm}$, IQR: $11-18 \mathrm{~mm}, P=.04)$. Plasma clot lysis time was comparable in patients with an abnormal and normal bleeding score (see Figure 3 and Table S4).

\section{4 | DISCUSSION}

This study reports on a cohort of 181 patients referred for analysis of a bleeding tendency in whom no major bleeding disorder was diagnosed. After routine haemostatic testing, $66 \%$ of patients remained undiagnosed and were classified as having bleeding of unknown cause (BUC). The other $34 \%$ of patients were diagnosed with a mild bleeding disorder (MBD).

We found that rotational thromboelastometry variables are within reference ranges in BUC patients and do not differ from healthy controls and MBD patients. Our results are in line with those recently described by Wieland Greguare-Sander et $\mathrm{al}^{25}$ and support their conclusion that there is no support for the additive value of rotational thromboelastometry for screening and diagnosing patients with a 
TABLE 2 Haemostatic variables in different patient groups and healthy controls

\begin{tabular}{|c|c|c|c|c|c|c|c|c|}
\hline & $N^{a}$ & BUC & $\mathrm{N}^{\mathrm{a}}$ & $\mathrm{HC}$ & $P^{c}$ & $\mathrm{~N}^{\mathrm{a}}$ & MBD & $P^{d}$ \\
\hline Haemoglobin, mmol/L & $61^{\mathrm{b}}$ & $8.1[7.9-8.8]$ & n.a. & & n.s. & $47^{b}$ & $8.7[8.2-9.1]$ & .006 \\
\hline Platelet count, $10^{9} / \mathrm{L}$ & $59^{\mathrm{b}}$ & 276 [229-328] & n.a. & & n.s. & $49^{\mathrm{b}}$ & 238 [207-278] & .012 \\
\hline $\mathrm{PT}, \mathrm{sec}$ & 116 & $11.9[11.4-12.5]$ & 76 & $11.9[11.5-12.6]$ & n.s. & 56 & $11.7[11.4-12.7]$ & n.s. \\
\hline \multirow[t]{2}{*}{ APTT, sec } & 121 & $25[23-26]$ & 76 & $25[23-26]$ & n.s. & 60 & 26 [24-27] & .010 \\
\hline & $63^{b}$ & $25[24-26]$ & n.a. & & n.s. & $51^{\mathrm{b}}$ & $26[24-27]$ & .026 \\
\hline \multirow[t]{2}{*}{ PFA, sec } & 117 & 137 [118-160] & 76 & 136 [109-157] & n.s. & 52 & $151[126-176]$ & n.s. \\
\hline & $63^{b}$ & 137 [118-159] & n.a. & & n.s. & $44^{\mathrm{b}}$ & 151 [123-173] & n.s. \\
\hline VWF:Ag, U/mL & $64^{b}$ & $0.91[0.73-1.18]$ & n.a. & & n.a. & $50^{\mathrm{b}}$ & $0.79[0.79-1.02]$ & .003 \\
\hline \multirow[t]{2}{*}{ VWF:Act, U/mL } & 121 & $0.89[0.75-1.22]$ & 76 & $0.93[0.77-1.24]$ & n.s. & 59 & $0.74[0.54-1.02]$ & .001 \\
\hline & $65^{\mathrm{b}}$ & $0.89[0.76-1.12]$ & n.a. & & n.s. & $50^{\mathrm{b}}$ & $0.79[0.54-1.04]$ & .026 \\
\hline \multirow[t]{2}{*}{ VWF:CB, U/mL } & 120 & $0.87[0.70-1.09]$ & n.a. & n.a. & n.a. & 59 & $0.69[0.48-0.96]$ & .000 \\
\hline & $64^{b}$ & 0.84 [0.70-1.07] & n.a. & & n.a. & $50^{\mathrm{b}}$ & $0.70[0.52-0.97]$ & .002 \\
\hline \multirow[t]{2}{*}{ FVIII:C, U/mL } & 121 & $1.20[1.05-1.40]$ & 75 & $1.23[1.13-1.53]$ & n.s. & 59 & $1.06[0.79-1.21]$ & .000 \\
\hline & $65^{\mathrm{b}}$ & $1.18[1.07-1.34]$ & n.a. & & n.s. & $50^{b}$ & $1.08[0.84-1.22]$ & .001 \\
\hline \multirow[t]{2}{*}{ FVII:C, U/mL } & 46 & $0.88[0.78-1.10]$ & 40 & $0.98[0.80-1.16]$ & n.s. & 26 & $0.91[0.74-1.13]$ & n.s. \\
\hline & $26^{\mathrm{b}}$ & $0.86[0.69-1.16]$ & n.a. & & n.s. & $22^{b}$ & $0.91[0.73-1.13]$ & n.s. \\
\hline \multirow[t]{2}{*}{ Alpha-2-antiplasmin, $\mathrm{U} / \mathrm{mL}$} & 88 & $1.15[1.05-1.21]$ & n.a. & n.a. & n.a. & 42 & $1.15[1.04-1.19]$ & n.s. \\
\hline & $53^{b}$ & 1.15 [1.09-1.22] & n.a. & & n.a. & $37^{\mathrm{b}}$ & 1.14 [1.03-1.19] & n.s. \\
\hline
\end{tabular}

Note: Data are shown as median and interquartile range [25th-75th percentile].

Abbreviations: aPTT: activated partial thromboplastin time; BUC: bleeding of unknown cause; FIX:C: factor IX activity; FVII:C factor VII activity; FVIII:C: factor VIII activity; FXI:C: factor XI activity; FXIII:C: factor XIII activity; HC: healthy controls; MBD: mild bleeding disorder; n.a.: not applicable; n.s.: nonsignificant; PFA: platelet function analyzer; PT: prothrombin time; VWF:Act: von Willebrand factor activity; VWF:Ag: von Willebrand factor antigen; VWF:CB: von Willebrand factor collagen binding.

${ }^{\mathrm{a} B a s e d}$ on available data.

${ }^{\mathrm{b}} \mathrm{No}$ of patients with abnormal bleeding score.

${ }^{\mathrm{C} C}$ Comparison of BUC patients and healthy controls.

${ }^{\mathrm{d} C}$ Comparison of BUC patients and patients with a MBD.

(mild) bleeding tendency. Thrombin generation has been applied regularly to investigate bleeding risk in patients with a bleeding disorder. $^{26,27}$ In this study, besides a significant longer lag time in BUC patients, thrombin generation parameters did not differ between patients with BUC and healthy controls, as also shown in previous studies. ${ }^{28,29}$ This finding was, however, in contrast with recently published data, ${ }^{24}$ in which all the TG variables in BUC patients were found to be significantly different from healthy controls. Patients with MBDs did show a significantly impaired thrombin generation, with a decreased endogenous thrombin potential (ETP) and peak height. This finding is remarkable, however, as the used thrombin generation is a reflection of secondary haemostasis, and most patients in the MBD group are diagnosed with a disorder of primary haemostasis. Also, no evidence was found supporting a systemic hyperfibrinolytic capacity in BUC patients. In contrast, we found that clot lysis time was significantly prolonged in BUC patients compared to healthy controls, in line with 

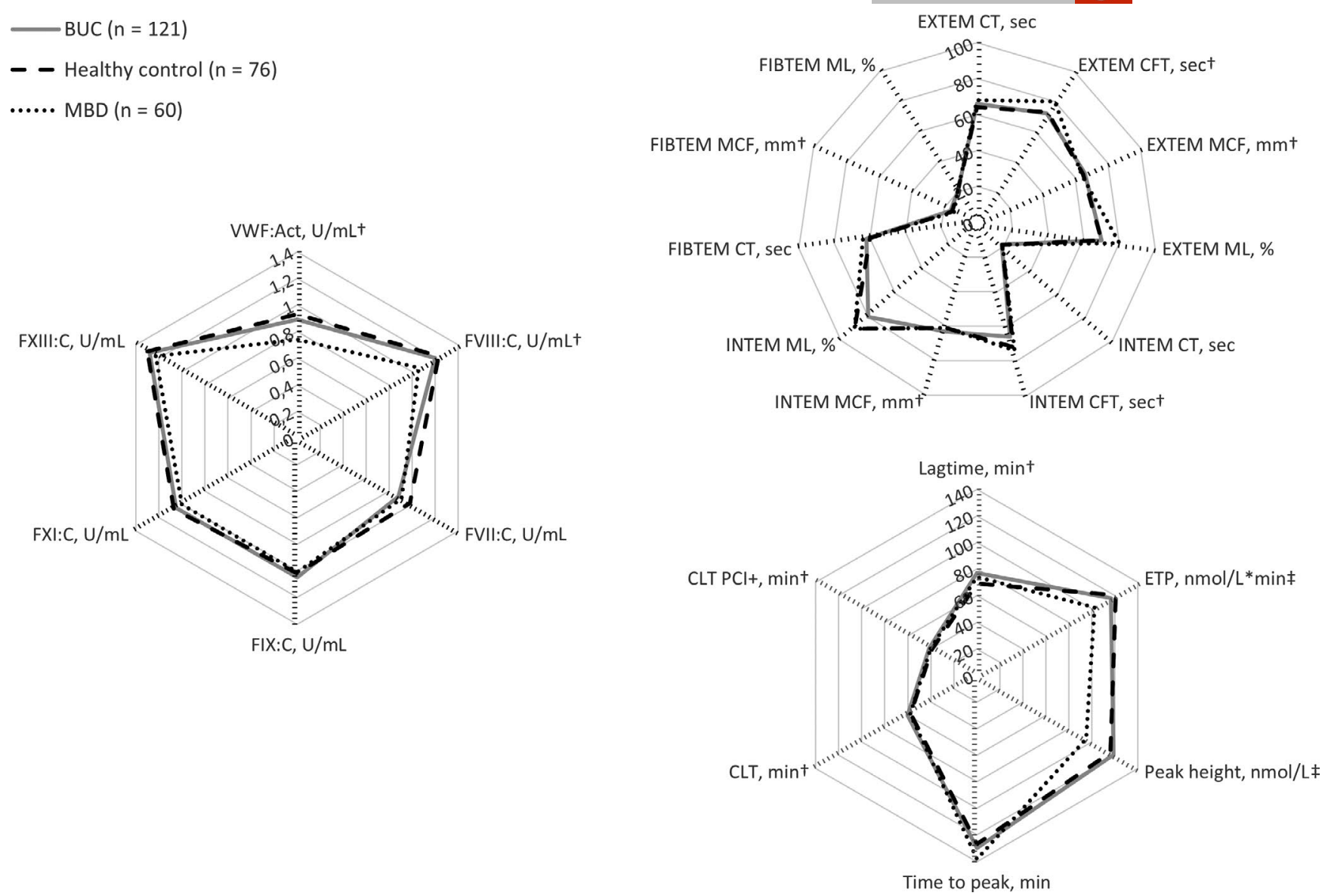

FIGURE 2 Haemostatic, thromboelastometry, thrombin generation and plasma clot lysis assay variables in patients and healthy controls. EXTEM ML $\times 10$; INTEM CT/10; INTEM ML × 10; FIBTEM ML $\times 10$; Lag time $\times 10$; ETP/10; Time to peak $\times 10$. BUC, bleeding of unknown cause; CFT, clot formation time; CLT, clot lysis time; CT, clotting time; ETP, endogenous thrombin potential; FIX:C, factor IX activity; FVII:C, factor VII activity; FVIII:C, factor VIII activity; FXI:C, factor XI activity; FXIII:C, factor XIII activity; MBD, mild bleeding disorder; MCF, maximum clot firmness; $\mathrm{ML}$, maximum lysis; $\mathrm{PCl}$, potato carboxypeptidase inhibitor; $\mathrm{VWF}$, von Willebrand factor. $\dagger P<.05$, BUC patients compared to healthy controls. $\ddagger P<.05$, BUC patients compared to MBD patients

previously published data, ${ }^{30,31}$ hereby carefully rejecting hyperfibrinolysis as underlying pathophysiological mechanism for BUC.

Several studies have shown that between $47 \%$ and $69 \%$ of patients will remain undiagnosed after extensive and repeated laboratory testing. ${ }^{1,4}$ When no laboratory abnormalities are found, the medical history and a bleeding score are important tools for physicians. ${ }^{6,32}$ However, the ISTH-BAT has shown to only have a limited role, as a normal score was present in $44 \%$ of BUC patients and in $21 \%$ of MBD patients. Therefore, a BAT should only serve as one of the many diagnostic tools available in the diagnostic work-up of these patients.

We confirm one of the main findings by Gebhart et al, namely, that the majority of patients being referred for bleeding symptoms is female (>80\%) and that more women than men are categorized with BUC, hereby possible affirming that there is a sex-related difference in BUC rate. ${ }^{1}$ Women have a higher chance of manifest bleeding due to menstrual cycle and women's ability for child birth. Other mechanisms however, such as the influence of female hormones on skin and muscle possibly leading to easy bruising, are still largely unknown. ${ }^{33}$ We also showed that patients with BUC were significantly older than patients with a diagnosed MBD. It has been shown that several haemostatic factors increase with age. ${ }^{34}$ This may explain that no abnormalities were found in this 'older' subgroup at time of analysis. Also, the role of comorbidities can be more pronounced in an older population, for example the influence of age and comorbidities on skin and vessels, possibly causing easy bruising or perioperative bleeding. ${ }^{35}$ To our knowledge, this is also one of the first studies to report BUC in adolescents, with a higher percentage of adolescents being diagnosed with a MBD than adults.

Our study has some limitations. First, one cannot exclude a possible referral bias for adolescents, with investigations possible being delayed or abandoned if the bleeding score was not very high. This might explain the increased rate of adolescents as well as the higher bleeding scores in the MBD patient group. We performed LTA for investigation of platelet function disorders. An influence of comedications on platelet function cannot be ruled out completely. For example, we did not exclude patients using selective serotonin reuptake inhibitors (SSRIs), which are shown to reduce platelet function. ${ }^{36,37}$ Due to the circadian rhythm of plasminogen activator inhibitor-1 (PAI-1), which inhibits 
Normal bleeding score $(n=137)$

- - Abnormal bleeding score $(n=117)$

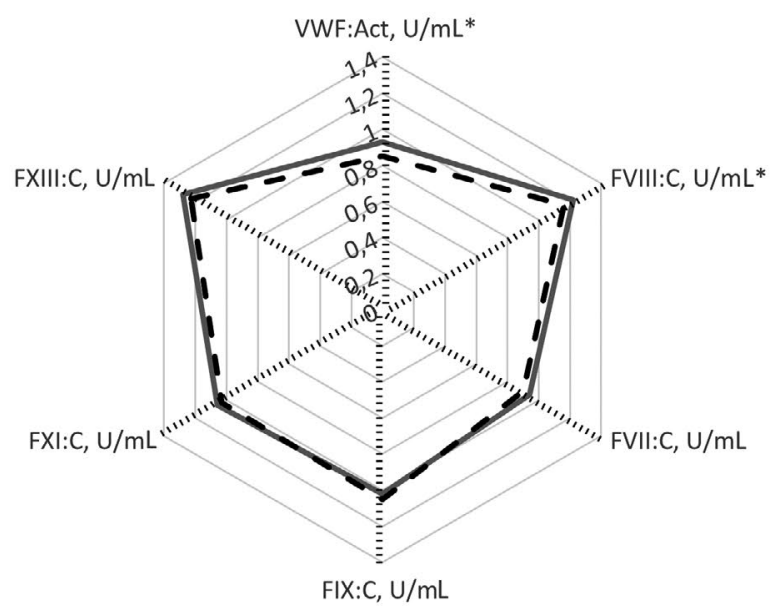

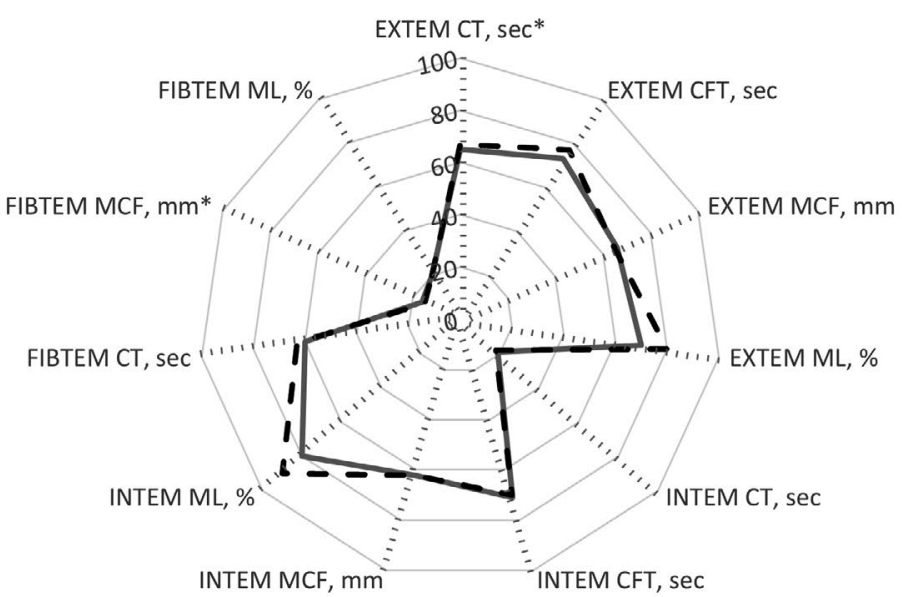

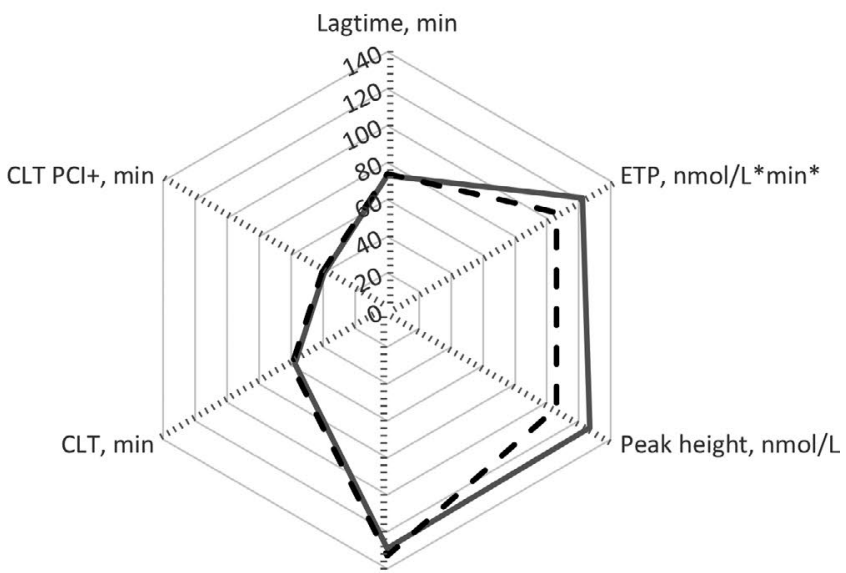

Time to peak, $\mathrm{min}$

FIGURE 3 Haemostatic, thromboelastometry, thrombin generation and plasma clot lysis assay variables based on bleeding score. EXTEM ML × 10; INTEM CT/10; INTEM ML × 10; FIBTEM ML × 10; Lag time × 10; ETP/10; Time to peak $\times 10$. CFT, clot formation time; CLT, clot lysis time; CT, clotting time; ETP, endogenous thrombin potential; FIX:C, factor IX activity; FVII:C, factor VII activity; FVIII:C, factor VIII activity; FXI:C, factor XI activity; FXIII:C, factor XIII activity; MCF, maximum clot firmness; ML, maximum lysis; PCI, potato carboxypeptidase inhibitor; VWF, von Willebrand factor. ${ }^{*} P<.05$

fibrinolysis and increases in the morning, ${ }^{38}$ we attempted to collect blood for plasma clot lysis assay in the afternoon. Unfortunately, this was not always possible due to logistic reasons.

Additional studies on patients without a clear diagnosis are required. In the near future, advanced techniques such as next-generation sequencing (NGS)-based gene panels ${ }^{39}$ or whole-exome sequencing (WES) ${ }^{40}$ may lead to discoveries of novel haemostatic modifiers. However, translating these results will provide a next challenge due to multi-interpretable and uncomprehensive findings such as variants of unknown significance (VUS).

\section{5 | CONCLUSION}

No major differences were found in thromboelastometry variables and thrombin generation in patients with bleeding of unknown cause (BUC), compared to healthy controls. BUC patients did have a significantly prolonged clot lysis time, possibly indicating an impaired or decreased fibrinolysis. In MBD patients, an impaired thrombin generation was found. At this point, however, we do not recommend implementation of thromboelastometry, measurement of thrombin generation and measurement of plasma clot lysis time in the diagnostic process of patients with bleeding of unknown cause.

\section{ACKNOWLEDGEMENTS}

The authors thank all participating patients and controls. The authors also thank the involved physicians of the Department of Haematology for their work on including patients and the staff of the diagnostic haemostasis laboratory for their help in processing samples, with special thanks to D.Priem. This study, also known as the Crescendo study (The Clinical Relevance and Significance of New Diagnostic Options-in patients with unexplained bleeding), is supported by research funding from Stichting Fonds NutsOhra.

\section{DISCLOSURES}

$\mathrm{MH}$ Cnossen has received grants from governmental research institutes such as Dutch Research Institute (NWO), ZonMW and 
Innovation fund, and unrestricted investigator initiated research grants as well as educational and travel funding from the following companies over the years: Pfizer, Baxter/ Baxalta/ Shire, Bayer Schering Pharma, CSL Behring, Sobi Biogen, Novo Nordisk, Novartis and Nordic Pharma, and has served as a member on steering boards of Roche and Bayer. All grants, awards and fees go to the institution. FWG Leebeek has received research funding by CSL Behring, Takeda/Shire and uniQure. He is consultant for uniQure and Takeda, for which fees go the university. He is DSMB member for a study by Roche. MJHA Kruip has received funding for research outside this work from Bayer, Pfizer, Daiichi Sankyo, Boehringer Ingelheim and ZonMW. MJHA Kruip has received speakers fee from Bayer. All other authors state that they had no interests which might be perceived as posing a conflict or bias. CSB Veen, MH Cnossen, MPM de Maat, FWG Leebeek and MJHA Kruip designed the study; CSB Veen and EH Huisman collected data; CSB Veen, DC Rljken, MPM de Maat and MJHA Kruip designed experiments, CSB Veen and R Kom-Gortat performed experiments; CSB Veen performed statistical analyses; CSB Veen, EH Huisman, DC Rijken, MPM de Maat and MJHA Kruip interpreted data; and CSB Veen and MJHA Kruip wrote the manuscript. All authors critically revised the manuscript, agreed with its content and approved submission.

\section{ORCID}

Caroline S. B. Veen (iD https://orcid.org/0000-0002-7671-9308

Frank W. G. Leebeek iD https://orcid.org/0000-0001-5677-1371

Marieke J. H. A. Kruip iD https://orcid.org/0000-0002-0265-4871

\section{REFERENCES}

1. Gebhart J, Hofer S, Panzer S, et al. High proportion of patients with bleeding of unknown cause in persons with a mild-to-moderate bleeding tendency: Results from the Vienna Bleeding Biobank (VIBB). Haemophilia. 2018;24(3):405-413.

2. Mauer AC, Khazanov NA, Levenkova N, et al. Impact of sex, age, race, ethnicity and aspirin use on bleeding symptoms in healthy adults. J Thromb Haemost. 2011;9(1):100-108.

3. Sadler JE. Low von Willebrand factor: sometimes a risk factor and sometimes a disease. Hematology Am Soc Hematol Educ Program. 2009;106-12.

4. Quiroga T, Mezzano D. Is my patient a bleeder? A diagnostic framework for mild bleeding disorders. Hematology Am Soc Hematol Educ Program. 2012;2012:466-474.

5. Mezzano D, Quiroga T. Diagnostic challenges of inherited mild bleeding disorders: a bait for poorly explored clinical and basic research. J Thromb Haemost. 2019;17(2):257-270.

6. Boender J, Kruip MJ, Leebeek FW. A diagnostic approach to mild bleeding disorders. J Thromb Haemost. 2016;14(8):1507-1516.

7. Obaji S, Alikhan R, Rayment R, Carter P, Macartney N, Collins P. Unclassified bleeding disorders: outcome of haemostatic challenges following tranexamic acid and/or desmopressin. Haemophilia. 2016;22(2):285-291.

8. Nair SC, Dargaud Y, Chitlur M, Srivastava A. Tests of global haemostasis and their applications in bleeding disorders. Haemophilia. 2010;16(Suppl 5):85-92.

9. Nogami K. The utility of thromboelastography in inherited and acquired bleeding disorders. Br J Haematol. 2016;174(4):503-514.
10. Hemker HC, Al Dieri R, De Smedt E, Beguin S. Thrombin generation, a function test of the haemostatic-thrombotic system. Thromb Haemost. 2006;96(5):553-561.

11. Antovic A, Mikovic D, Elezovic I, Zabczyk M, Hutenby K, Antovic JP. Improvement of fibrin clot structure after factor VIII injection in haemophilia A patients treated on demand. Thromb Haemost. 2014;111(4):656-661.

12. Wolberg AS, Allen GA, Monroe DM, Hedner U, Roberts HR, Hoffman M. High dose factor VIla improves clot structure and stability in a model of haemophilia B. Br J Haematol. 2005;131(5):645-655.

13. Rodeghiero F, Castaman G, Tosetto A, et al. The discriminant power of bleeding history for the diagnosis of type 1 von Willebrand disease: an international, multicenter study. J Thromb Haemost. 2005;3(12):2619-2626.

14. Elbatarny M, Mollah S, Grabell J, et al. Normal range of bleeding scores for the ISTH-BAT: adult and pediatric data from the merging project. Haemophilia. 2014;20(6):831-835.

15. Loeffen R, Kleinegris MC, Loubele ST, et al. Preanalytic variables of thrombin generation: towards a standard procedure and validation of the method. J Thromb Haemost. 2012;10(12):2544-2554.

16. Hemker HC, Giesen PL, Ramjee M, Wagenvoord R, Beguin S. The thrombogram: monitoring thrombin generation in platelet-rich plasma. Thromb Haemost. 2000;83(4):589-591.

17. Lisman T, de Groot PG, Meijers JC, Rosendaal FR. Reduced plasma fibrinolytic potential is a risk factor for venous thrombosis. Blood. 2005;105(3):1102-1105

18. Talens S, Malfliet JJ, Rudez G, etal. Biological variation in tPA-induced plasma clot lysis time. Thromb Haemost. 2012;108(4):640-646.

19. CLSI. Defining, establishing, and verifying reference intervals in the clinical laboratory; approved guidline (Third ed.). Wayne, PA: CLSI; 2008.

20. Geffre A, Concordet D, Braun JP, Trumel C. Reference value advisor: a new freeware set of macroinstructions to calculate reference intervals with Microsoft excel. Vet Clin Pathol. 2011;40(1):107-112.

21. Leebeek FW, Eikenboom JC. Von Willebrand's disease. N Engl J Med. 2016;375(21):2067-2080.

22. Gresele P, Subcommittee on Platelet Physiology of the International Society on T, Hemostasis. Diagnosis of inherited platelet function disorders: guidance from the SSC of the ISTH. J Thromb Haemost. 2015;13(2):314-322.

23. Mumford AD, Ackroyd S, Alikhan R, et al. Guideline for the diagnosis and management of the rare coagulation disorders: a United Kingdom Haemophilia Centre Doctors' Organization guideline on behalf of the British Committee for Standards in Haematology. Br J Haematol. 2014;167(3):304-326.

24. Hofer S, Ay C, Rejto J, et al. Thrombin-generating potential, plasma clot formation, and clot lysis are impaired in patients with bleeding of unknown cause. J Thromb Haemost. 2019;17(9):1478-1488.

25. Wieland Greguare-Sander A, Wuillemin WA, Nagler M. Thromboelastometry as a diagnostic tool in mild bleeding disorders: a prospective cohort study. Eur J Anaesthesiol. 2019;36(6):457-465.

26. Zekavat OR, Haghpanah S, Dehghani J, Afrasiabi A, Peyvandi F, Karimi M. Comparison of thrombin generation assay with conventional coagulation tests in evaluation of bleeding risk in patients with rare bleeding disorders. Clin Appl Thromb Hemost. 2014;20(6):637-644.

27. Van Geffen M, Menegatti M, Loof A, et al. Retrospective evaluation of bleeding tendency and simultaneous thrombin and plasmin generation in patients with rare bleeding disorders. Haemophilia. 2012;18(4):630-638.

28. Ay C, Haselbock J, Laczkovics C, Koder S, Pabinger I. Thrombin generation in patients with a bleeding tendency of unknown origin. Ann Hematol. 2011;90(9):1099-1104. 
29. Quiroga T, Goycoolea M, Giesen PL, et al. Thrombin generation in platelet-poor plasma is normal in patients with hereditary mucocutaneous haemorrhages. Pathophysiol Haemost Thromb. 2003;33(1):30-35.

30. Vries MJA, Macrae F, Nelemans PJ, et al. Assessment and determinants of whole blood and plasma fibrinolysis in patients with mild bleeding symptoms. Thromb Res. 2019;174:88-94.

31. Gebhart J, Laczkovics C, Posch F, et al. Plasma clot properties in patients with a mild-to-moderate bleeding tendency of unknown cause. Ann Hematol. 2015;94(8):1301-1310.

32. Tosetto A, Castaman G, Rodeghiero F. Bleeders, bleeding rates, and bleeding score. J Thromb Haemost. 2013;11(Suppl 1):142-150.

33. Knol HM, Kemperman RF, Kluin-Nelemans HC, Mulder AB, Meijer K. Haemostatic variables during normal menstrual cycle. A systematic review. Thromb Haemost. 2012;107(1):22-29.

34. Sanders YV, Giezenaar MA, Laros-van Gorkom BA, et al. von Willebrand disease and aging: an evolving phenotype. J Thromb Haemost. 2014;12(7):1066-1075.

35. Atiq F, Meijer K, Eikenboom J, et al. Comorbidities associated with higher von Willebrand factor (VWF) levels may explain the age-related increase of VWF in von Willebrand disease. $\mathrm{Br} J$ Haematol. 2018;182(1):93-105.

36. Lopez-Vilchez I, Jerez-Dolz D, Diaz-Ricart M, et al. Escitalopram impairs thrombin-induced platelet response, cytoskeletal assembly and activation of associated signalling pathways. Thromb Haemost. 2017;117(12):2312-2321.

37. Tseng YL, Chiang ML, Huang TF, Su KP, Lane HY, Lai YC. A selective serotonin reuptake inhibitor, citalopram, inhibits collagen-induced platelet aggregation and activation. Thromb Res. 2010;126(6):517-523.

38. Scheer FA, Shea SA. Human circadian system causes a morning peak in prothrombotic plasminogen activator inhibitor-1 (PAI-1) independent of the sleep/wake cycle. Blood. 2014;123(4):590-593.

39. Simeoni I, Stephens JC, Hu F, et al. A high-throughput sequencing test for diagnosing inherited bleeding, thrombotic, and platelet disorders. Blood. 2016;127(23):2791-2803.

40. Leinoe $\mathrm{E}$, Zetterberg $\mathrm{E}$, Kinalis $\mathrm{S}$, et al. Application of whole-exome sequencing to direct the specific functional testing and diagnosis of rare inherited bleeding disorders in patients from the Oresund Region, Scandinavia. Br J Haematol. 2017;179(2):308-322.

\section{SUPPORTING INFORMATION}

Additional supporting information may be found online in the Supporting Information section.

How to cite this article: Veen CSB, Huisman EJ, Cnossen $\mathrm{MH}$, et al. Evaluation of thromboelastometry, thrombin generation and plasma clot lysis time in patients with bleeding of unknown cause: A prospective cohort study. Haemophilia. 2020;00:1-10. https://doi.org/10.1111/ $\underline{\text { hae.13991 }}$ 